\title{
Prescripción inapropiada de antifúngicos y la necesidad de programas para su uso racional
}

\author{
Juan Pablo Osorio Lombana1,2, Sonia Isabel Cuervo Maldonado, ${ }^{1,2,3}$ \\ María José López Mora² y Julio César Gómez Rincón",3
}

\section{Inappropriate antifungal prescription and the need for antifungal stewardship programs}

Invasive fungal disease (IFD) is a condition affecting immunosuppressed and critically ill patients. Recently there has been an increase in the amount of patients at risk for IFD, which implies an increase in the prescription of antifungal agents as prophylactic, pre-emptive or empiric therapy. Some studies evaluating appropriateness of antifungal prescription have shown that inappropriate formulations reach $72 \%$, exposing patients to side effects, pharmacological interactions and rising costs. Some groups have recommended many interventions to control and make a rational use of antimicrobials, into strategies known as "antimicrobial stewardship", these interventions are useful also for antifungal agents and it has been named "antifungal stewardship". Here we present a narrative review of the scientific literature showing published articles about appropriate use of antifungal agents and the experience of some centers after implementing antifungal stewardship programs.

Keywords: Invasive fungal disease; antifungal prescription; antimicrobial stewardship; antifungal stewardship.

Palabras clave: Enfermedad fúngica invasora; prescripción; antifúngicos; uso apropiado.

\section{Introducción}

$\mathrm{E}$ $\mathrm{n}$ la actualidad, varios factores han favorecido el aumento progresivo de la frecuencia de la enfermedad fúngica invasora (EFI), entre ellos la mayor expectativa de vida, la mayor prevalencia de enfermedades crónicas, mayor frecuencia de inmunodeficiencias secundarias debidas a una gran lista de enfermedades neoplásicas, auto-inflamatorias, el trasplante de órgano sólido (TOS) o de células progenitoras hematopoyéticas, o relacionada al uso de dispositivos médicos invasores. La EFI se asocia a altas tasas de mortalidad y a aumento de los costos en la atención de estos pacientes, lo que ha llevado a un mayor uso de antifúngicos a nivel hospitalario y ambulatorio. De manera global, se estima que $4 \%$ de las prescripciones de antimicrobianos al interior de los hospitales corresponde a antifúngicos, y varios estudios describen que el uso no apropiado de antifúngicos varía entre 7 y $74 \% \%^{1-5}$, resultados que dependen de los criterios de inclusión y de definición de EFI utilizados. Teniendo en cuenta que, como parte del control de la prescripción y de la diseminación de la resistencia antimicrobiana, se propone un programa de administración (stewardship) que optimice el manejo de los antimicrobianos, nos preguntamos si esta estrategia puede ser útil en el escenario de la EFI. El objetivo de esta revisión es describir el uso inapropiado de antifúngicos en el mundo y destacar las principales estrategias que han mostrado ser efectivas en el programa de uso apropiado de antimicrobianos, que pudieran emplearse para la formulación de antifúngicos.

\section{Métodos}

Se realizó una búsqueda en PubMed (base de datos de la Biblioteca Nacional de Medicina de los Institutos Nacionales de Salud de EUA) con los términos "antifungal stewardship", y artículos relacionados con el uso apropiado de antifúngicos utilizando los términos "antifungal use", "antifungal use appropriateness" y "antifungal prescription"- Además se revisaron algunas guías de práctica clínica con recomendaciones para el diseño de programas de uso racional de antimicrobianos, y sus recomendaciones para los antifúngicos. Se tomaron para la revisión artículos con información relevante respecto a la epidemiología de la EFI, el uso inapropiado de antifúngicos y el impacto de la implementación de programas de uso optimizado de antifúngicos.

\section{Resultados}

A continuación, se presentan los principales hallazgos descritos en la literatura médica sobre el impacto de la EFI y su relación con la formulación de los antifúngicos disponibles para su tratamiento.

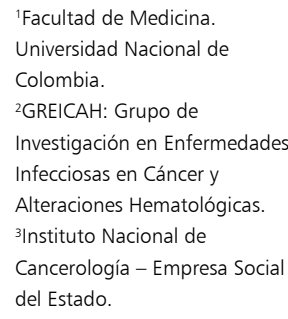

Los autores declaran no tener conflictos de interés.

Fuente de financiación: ninguna.

Recibido: 12 de agosto de 2018 Aceptado: 1 de abril de 2019

Correspondencia a: Juan Pablo Osorio Lombana jposoriol@unal.edu.co 


\section{El problema de la enfermedad fúngica invasora}

La EFI es una entidad que afecta a pacientes inmunocomprometidos (pacientes con neoplasias hematológicas, receptores de TOS como de trasplante de progenitores hematopoyéticos (TPH), con uso de corticoesteroides, o infección por VIH) y a pacientes críticamente enfermos, siendo una causa importante de morbilidad y mortalidad en estos grupos de pacientes ${ }^{6}$. La epidemiología de la EFI ha cambiado con el advenimiento de nuevos esquemas de quimioterapia, el uso de terapia biológica y las estrategias de profilaxis antifúngica en pacientes sometidos a inmunosupresión; la aproximación inicial al diagnóstico y manejo de la EFI obliga a establecer el riesgo individual del paciente teniendo en cuenta sus condiciones de base y la epidemiología local?

\section{Epidemiología de la EFI}

Se estima que la frecuencia de EFI en pacientes con neoplasias hematológicas es de aproximadamente 4,6\% (entre 2 y $49 \%$, según el centro que se considere); sin embargo, no es igual en todos los grupos de pacientes hematológicos. Los pacientes con leucemia aguda (frecuencia observada en 1 año: $12 \%$ ) y receptores de trasplante alogénico de progenitores hematopoyéticos (frecuencia observada en 1 año: 3,4-7,2\%) tienen mayor riesgo para desarrollar $\mathrm{EFI}^{8}$. En receptores de TPH, el riesgo de presentar EFI depende del tipo de trasplante, el origen de los progenitores hematopoyéticos, el desarrollo de enfermedad injerto contra hospedero y el uso de profilaxis $^{6,9}$. En las primeras descripciones, la etiología de la EFI fue mayoritariamente por Candida spp., pero debido al uso profiláctico de fluconazol en pacientes de alto riesgo, su incidencia ha disminuido, y en cambio se ha hecho más frecuente la EFI causada por hongos filamentosos ${ }^{6}$. La mortalidad de la EFI en pacientes con neoplasias hematológicas puede llegar a $60 \%$, con variación según la etiología, siendo más alta en mucormicosis que en aspergilosis invasora (AI) y que en candidiasis invasora $(\mathrm{CI})^{10,11}$.

En pacientes receptores de TOS se ha reportado una frecuencia de EFI en los 12 meses posteriores al trasplante de 3,1\% (0,7-23\%), la que varía de acuerdo al órgano trasplantado, siendo mayor en trasplante de intestino $(11,6 \%)$ y corazón-pulmón $(8,6 \%)$, que en hígado $(3,4 \%)$ o riñón $(1,3 \%)$. La mortalidad por EFI en receptores de TOS es de 27 a $30 \%$, siendo mayor en pacientes con trasplante de hígado ${ }^{10,12,13}$. Los principales agentes etiológicos responsables de EFI en este grupo de pacientes son Candida spp. (2-4\%), seguida de Aspergillus spp $(0,1-2,4 \%)$, y menos frecuentemente infecciones por Cryptococcus sp, Fusarium sp, Scedosporium sp y mucormicosis ${ }^{13}$.

En el paciente críticamente enfermo, la EFI constituye también una carga importante de morbilidad y mortali- dad, siendo la candidiasis invasora la infección fúngica más frecuente, dada principalmente por infección del torrente sanguíneo. Como factores de riesgo se identifican la estancia prolongada en cuidados intensivos, el uso de antimicrobianos de amplio espectro, el tener acceso venoso central, recibir nutrición parenteral total, la colonización multifocal por Candida spp., haber sido sometido a cirugía y algún estado de inmunosupresión. La aspergilosis invasora en el paciente crítico, en ausencia neutropenia, cáncer y trasplante, puede afectar a pacientes con uso de corticoesteroides o con enfermedad pulmonar obstructiva crónica (EPOC) con una mortalidad entre 80 y $97 \%{ }^{14}$.

\section{Diagnóstico}

El diagnóstico de la EFI es complejo teniendo en cuenta que los pacientes usualmente cursan con condiciones de riesgo que dificultan los procedimientos de diagnóstico invasores, tales como lavado broncoalveolar, biopsia y cultivos de tejido, (citopenias, estado crítico), el rendimiento de las pruebas de laboratorio no es óptimo y la recuperación microbiológica de los agentes no es fácil. Se establece entonces el diagnóstico mediante un constructo de criterios clínicos, factores de riesgo del paciente, microbiológicos e histopatológicos. Con el fin de estandarizar definiciones, de cara a la investigación de pruebas diagnósticas y de estrategias terapéuticas, se categoriza la EFI en: posible, probable o probada, de acuerdo a la clasificación propuesta por la European Organization for Research and Treatment of Cancer y el National Institute of Allergy and Infectious Diseases Mycoses Study Group (EORTC/MSG) ${ }^{15}$ (Tablas 1 y 2).

Se establece el diagnóstico de EFI probable en pacientes que tengan factor del hospedero + criterio clínico + criterio micológico. Se clasifica como EFI posible, los pacientes que presenten factor del hospedero + criterio clínico, sin tener criterio micológico. El diagnóstico de EFI probada requiere la recuperación del hongo en cultivo tomado del sitio afectado o en sangre, o la demostración histopatológica o microscópica de estructuras fúngicas ${ }^{15}$ (Tabla 2). Es importante anotar que dicha calificación no implica una decisión de tratar o no tratar desde el punto de vista clínico.

Las dificultades en el diagnóstico de la EFI (que incluyen la identificación tardía del cuadro y el pobre rendimiento diagnóstico de pruebas de laboratorio), su alta mortalidad, el aumento de complicaciones con el inicio tardío de terapia efectiva ${ }^{16,17}$, y la disponibilidad de agentes antifúngicos de amplio espectro con menores efectos adversos, llevan a que la mayoría de pacientes con riesgo de EFI reciban tratamiento antifúngico de forma empírica por el equipo tratante, sin que necesariamente esté orientada por expertos en infecciones fúngicas ${ }^{18}$. 


\begin{tabular}{|c|c|c|}
\hline & Hongos filamentosos & Levaduras \\
\hline Microscopía & $\begin{array}{l}\text { Estudio histopatológico, citopatológico o directo, en muestra obtenida } \\
\text { por biopsia o aspiración con aguja fina, con hifas o formas fúngicas } \\
\text { melanínicas, acompañadas de evidencia de daño tisular }\end{array}$ & $\begin{array}{l}\text { Estudio histopatológico, citopatológico o directo, en muestra obtenida } \\
\text { por biopsia o aspiración con aguja fina, de sitio normalmente estéril, } \\
\text { que muestre levaduras }\end{array}$ \\
\hline Cultivo & $\begin{array}{l}\text { Recuperación de hongo micelial o melanínico por cultivo de muestra } \\
\text { obtenida por un procedimiento estéril de un sitio normalmente estéril } \\
\text { o de sitio clínica o radiológicamente anormal consistente con un pro- } \\
\text { ceso infeccioso, excluyendo lavado bronco-alveolar, cavidad sinusal } \\
\text { craneal y orina. }\end{array}$ & $\begin{array}{l}\text { Recuperación de levadura por cultivo de muestra obtenida por proce- } \\
\text { dimiento estéril de un sitio normalmente estéril o clínica o radiológica- } \\
\text { mente anormal consistente con proceso infeccioso }\end{array}$ \\
\hline Hemocultivo & $\begin{array}{l}\text { Hemocultivo positivo con crecimiento de un hongo micelial en el } \\
\text { contexto de una enfermedad infecciosa compatible. El crecimiento de } \\
\text { Aspergillus spp. se considera contaminación }\end{array}$ & $\begin{array}{l}\text { Hemocultivo positivo con crecimiento de levaduras o de hongos le- } \\
\text { vaduriformes }\end{array}$ \\
\hline Análisis serológico & No aplica & $\begin{array}{l}\text { Antígeno para Cryptococcus spp. positivo en líquido cefalorraquídeo } \\
\text { indica criptococosis }\end{array}$ \\
\hline
\end{tabular}

\section{Tabla 2. Criterios EORTC/MSG para el diagnóstico de la EFI probable y posible ${ }^{15}$}

Factores del Historia reciente de neutropenia ( $<500$ céls $/ \mathrm{mm}^{3}$ por $>10$ días) relacionada temporalmente con el inicio de la enfermedad fúngica

hospedero Receptor de trasplante alogénico de progenitores hematopoyéticos

Uso prolongado de corticosteroides a dosis de al menos 0,3 mg/kg/día de prednisona o equivalente por más de tres semanas

Tratamiento con inmunosupresores de linfocitos T (ciclosporina, bloqueadores de FNT- $\alpha$, anticuerpos monoclonales (alemtuzumab) o análogos de nucleósidos en los últimos 90 días

Inmunodeficiencia hereditaria grave (enfermedad granulomatosa crónica o inmunodeficiencia combinada grave)

Criterios Enfermedad de tracto respiratorio inferior

clínicos

Traqueobronquitis

Sino-nasal

Sistema nervioso central

Candidiasis diseminada (presencia de signos y síntomas consistentes con sepsis, indican enfermedad diseminada aguda)

Criterios Prueba directa (citología, microscopía directa micológicos o cultivo)
Presencia de uno de los siguientes signos en TAC: Lesión densa bien circunscrita, con o sin signo de halo, signo de "aire creciente", cavitación

Úlcera, nódulo, pseudomembrana, placa o escara traqueo-bronquial en broncoscopia

Imagen sugerente de sinusitis, con al menos uno de los siguientes: dolor localizado agudo (incluye dolor irradiado al ojo), úlcera nasal con escara negra, extensión desde los senos paranasales a través de las paredes óseas (incluyendo hacia la órbita)

Uno de los siguientes: lesión focal en imagen, realce meníngeo en TAC o IRM

Al menos uno de los siguientes, luego de un episodio de candidemia en las dos semanas previas: abscesos pequeños en forma de diana en hígado o bazo, exudados retinianos progresivos en el examen oftalmológico

Hongo micelial en esputo, lavado bronco-alveolar, cepillado bronquial, o muestra de aspirado sinusal, indicado por uno de los siguientes: presencia de elementos fúngicos, recuperación en cultivo de hongo micelial (Aspergillus spp., Fusarium spp., Zygomicetos o Scedosporium spp.)

Pruebas indirectas (detección de antígenos o elementos de la pared)
Aspergilosis: antígeno galactomanano en plasma, suero, lavado bronco-alveolar o líquido cefalorraquídeo EFI distinta a criptococosis o zigomicosis: $\beta$-D-glucano detectado en suero

\section{Prescripción inapropiada de antifúngicos}

La prescripción de antifúngicos constituye $4 \%$ de las formulaciones de antimicrobianos en algunos centros, se realiza predominantemente en servicios que atienden pacientes de alto riesgo para EFI como hematología, oncología y unidades de cuidado crítico ${ }^{2}$. Las indicacio- nes habituales para el uso de antifúngicos son la terapia dirigida, la terapia empírica, la terapia anticipada y la profilaxis.

Algunos centros han evaluado la prescripción de antifúngicos, encontrando diferentes frecuencias de uso no apropiado (Tabla 3). Las principales causas detectadas han 


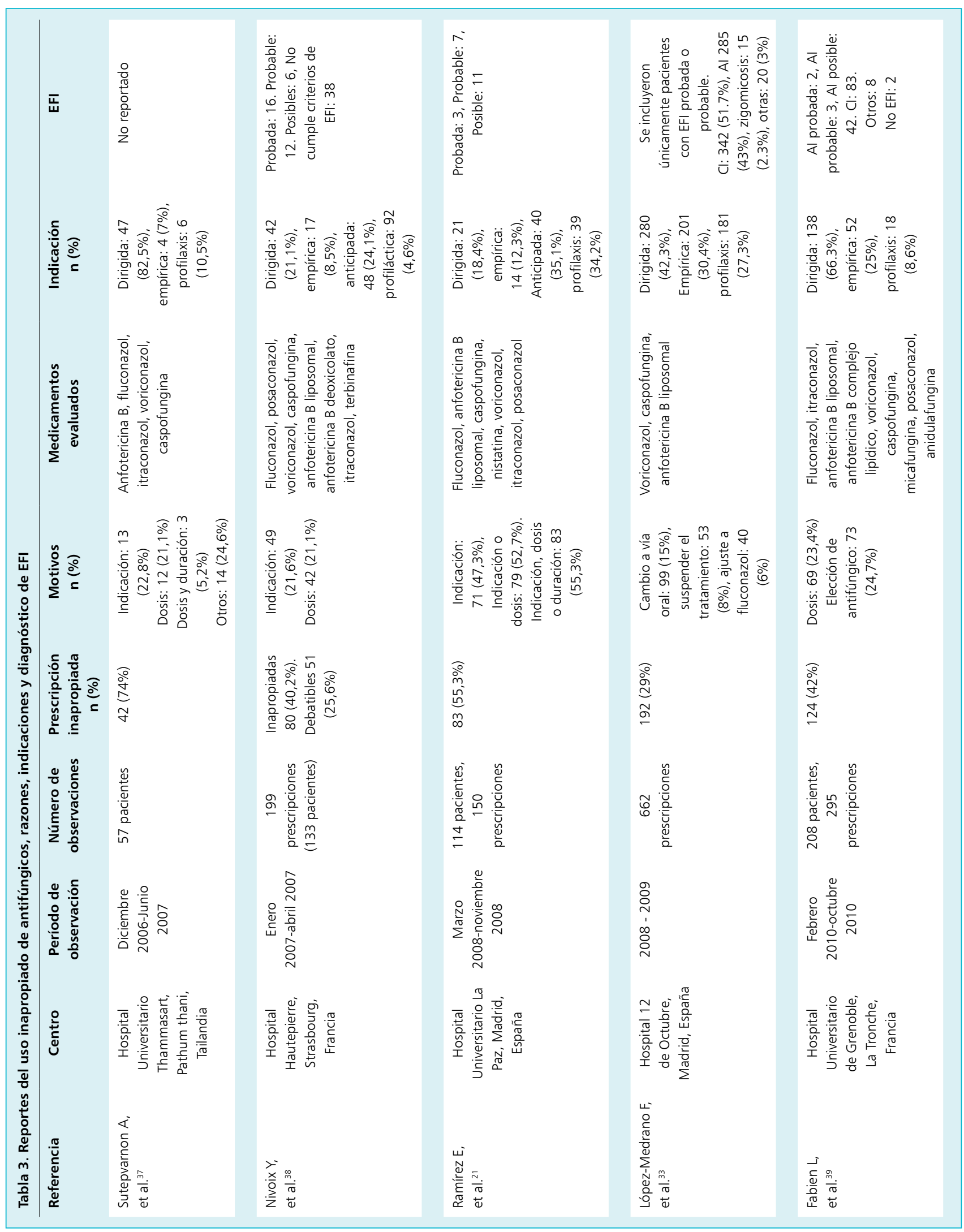




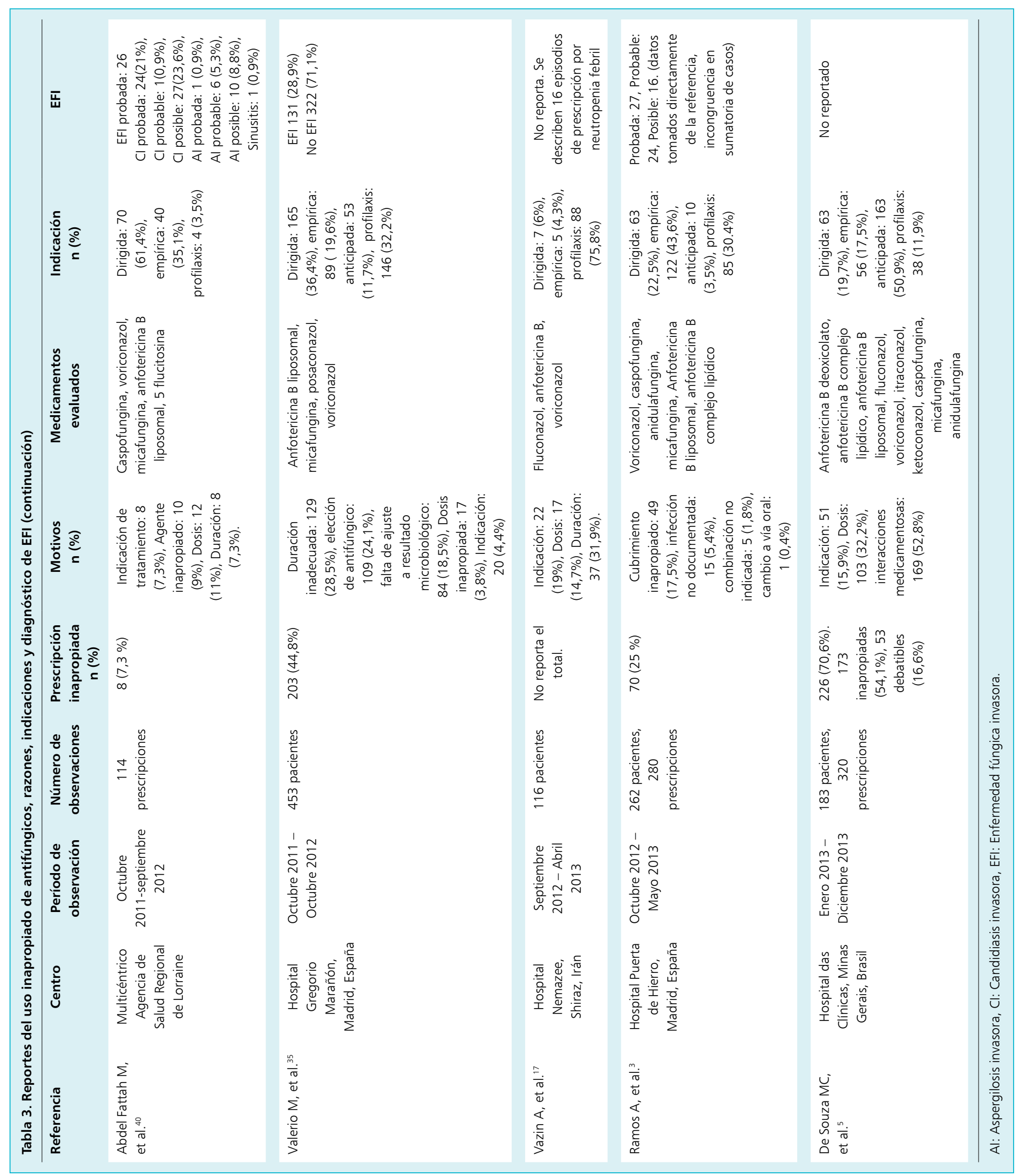


sido: falta de indicación de la terapia, dosis no ajustada, desconocimiento de la epidemiología local, no ajuste de la terapia empírica, no cambios de la terapia parenteral a la oral, entre otras. La prescripción inapropiada de algún agente antifúngico expone a los pacientes a efectos adversos, riesgo de interacciones medicamentosas, aumenta los costos en la atención y contribuye al incremento en la resistencia a antifúngicos o a la aparición de EFI de brecha o EFI emergentes, conocidas en inglés como breakthrough infections ${ }^{1,3}$.

En la Tabla 3 se presentan algunas descripciones reportadas en la literatura médica sobre el uso no apropiado de antifúngicos en diferentes grupos de pacientes. Los reportes de uso inapropiado de antifúngicos y de terapia dirigida para la EFI muestran que la prescripción inapropiada de antifúngicos es frecuente, aunque las frecuencias son variables. El escenario clínico para el uso de antifúngicos es, en su mayoría, la EFI posible o probable, con una frecuencia baja de terapia dirigida.

Se requiere la implementación de medidas para optimizar la prescripción, mejorar las estrategias de diagnóstico y hacer el ajuste de la terapia, de acuerdo con los hallazgos de laboratorio. El primer paso para el diseño y la implementación de medidas que busquen el uso racional de antifúngicos, es conocer y cuantificar el consumo de estos medicamentos a nivel local.

\section{Descripción del uso institucional de antifúngicos}

Para conocer el consumo de antifúngicos de una institución existen diferentes indicadores. La cuantificación del uso de antimicrobianos, incluyendo los antifúngicos, tiene fines tanto descriptivos desde la perspectiva institucional (costos, consumo, compras), como de seguimiento para medir el impacto de estrategias que intervengan el uso de medicamentos. Los estimadores más usados son los siguientes: Días de Terapia (DOT, por su sigla en inglés) por 1.000 pacientes/día o por 1.000 ingresos, Dosis Diaria Definida (DDD) por 1.000 pacientes/día, y Duración de la Terapia (LOT, por su sigla en inglés) por ingresos o egresos o por 1.000 pacientes/día ${ }^{19}$.

El uso de antifúngicos en las instituciones es muy variable, depende no sólo de la cantidad de pacientes que atienden, sino de la presencia en ellos de factores de riesgo para EFI. En la literatura médica se encuentran algunos reportes de uso de antifúngicos por institución, que van desde 40 a 296 DDD/1.000 pacientes-día ${ }^{1}$, al igual que estudios de consumo regional, multicéntricos, como el reportado por Fondevilla y cols., mostrando un consumo de 25 a 30,6 DDD/1.000 pacientes-día ${ }^{20}$. El indicador de DDD es un indicador práctico recomendado por la Organización Mundial de la Salud; sin embargo, éste es un estimador que refleja el uso local y no siempre es comparable entre instituciones ${ }^{19,21}$.

\section{Programas para el uso racional de antifúngicos- “Antifungal stewardship"}

En los últimos años, el consumo mundial de antimicrobianos ha aumentado en $36 \%$, incluyendo antibacterianos como carbapenémicos y polimixinas ${ }^{22}$. Se estima que ha ocurrido algo similar con los antifúngicos, teniendo en cuenta el creciente número de pacientes en riesgo de desarrollar EFI, aunque el uso de antifúngicos ha recibido menor atención que el uso de antibacterianos con respecto al desarrollo de programas de optimización en uso de antimicrobianos (antimicrobial stewardship) ${ }^{20}$.

Un fenómeno que ha generado interés para el desarrollo de estos programas es la resistencia bacteriana, la que surge como resultado de la presión selectiva de los microorganismos frente a la exposición a los antibacterianos ${ }^{22}$. Con respecto a la resistencia en hongos y su relación con el uso de antifúngicos, algunos estudios han mostrado que el uso de fluconazol se relaciona con la emergencia de especies de Candida no-albicans y mayor riesgo de resistencia a azoles ${ }^{23}$, mientras que el uso de equinocandinas se ha relacionado con mayor probabilidad de selección de otros hongos que son resistentes a la mayoría de los antifúngicos disponibles. Candida spp., es un colonizante de mucosas y piel, sobre el que es más probable que el uso médico de azoles induzca presión selectiva. Los hongos miceliales (especialmente Aspergillus spp.), de manera diferente a lo que ocurre en Candida spp., tienen un nicho ecológico ambiental, lo que los hace menos susceptibles a la presión del uso médico de antifúngicos en humanos. Se ha reportado 3,2\% de resistencia a azoles en Aspergillus fumigatus ${ }^{24}$, y en un estudio de muestras de suelo en Colombia resistencia de $9,3 \%{ }^{25}$. Tanto el aislamiento microbiológico como la realización de pruebas de sensibilidad a antifúngicos en hongos miceliales son complejos, lo que dificulta el estudio de la prevalencia de resistencia en este grupo de microorganismos. Sin embargo, considerando el nicho ecológico de Aspergillus spp., es probable que el fenómeno de resistencia y presión selectiva se presente más con el uso de antifúngicos en agricultura que con el uso hospitalario de antifúngicos ${ }^{10,24}$.

\section{Programas de uso óptimo de antimicrobianos}

En la última década, diferentes organizaciones de orden regional o local han emitido recomendaciones para la elaboración y puesta en marcha de programas para el uso óptimo de antimicrobianos en instituciones hospitalarias $^{26-28}$. Estos programas consisten en grupos de intervenciones diseñadas para disminuir el uso inapropiado de antimicrobianos ( $\mathrm{p}$ ej. mejorar la selección del medicamento, dosis, duración y vía de administración), con el objetivo de mejorar los desenlaces clínicos, evitar toxicidad e interacciones medicamentosas, disminuir los costos, y evitar la selección de microorganismos resistentes ${ }^{29}$. 
Dentro de los elementos fundamentales recomendados para establecer los programas para el uso racional de antimicrobianos se encuentran los siguientes ${ }^{27,28,30}$ :

- Educación: es el primer paso para el establecimiento de programas de uso racional de antimicrobianos. Se debe dirigir tanto a prescriptores como a personal en formación, se logra de mejor manera con el uso de escenarios clínicos que con actividades didácticas como clases o conferencias. De acuerdo con los recursos disponibles, se pueden realizar actividades presenciales o virtuales, se debe brindar retroalimentación desde el grupo de uso racional hacia los prescriptores y hacerlos partícipes de la toma de decisiones lo que puede mejorar la aceptación de las recomendaciones por el grupo tratante.

- Equipo interdisciplinario: se debe incluir a personal con conocimiento en el manejo de antimicrobianos: médico con entrenamiento en enfermedades infecciosas, personal de farmacia (químico farmacéutico o farmacólogo clínico), profesional de control de infecciones o epidemiología, laboratorio clínico, experto en tecnologías de información, personal administrativo (administración hospitalaria), y comité de compras ${ }^{29}$.

- Desarrollo de guías de práctica clínica: es la piedra angular del uso racional de antimicrobianos; se deben desarrollar desde el nivel local con base en revisiones basadas en la evidencia y estar ajustadas a los recursos y a la epidemiología de las instituciones. Se debe promover tanto su divulgación, como su implementación. La medida del cumplimiento de las recomendaciones es útil como un indicador que sirve para cuantificar el uso apropiado de los antimicrobianos y el impacto de intervenciones del programa de uso racional de antimicrobianos.

- Mejoría diagnóstica: teniendo en cuenta que el resultado de las pruebas de microbiología convencional es usualmente tardío, se propone considerar la realización de pruebas diagnósticas especiales, como el uso de biomarcadores, pruebas moleculares o serológicas, como alternativa para la identificación de microorganismos ${ }^{31}$. Es fundamental la articulación entre el laboratorio de microbiología y los médicos clínicos para tener reportes oportunos (incluso de pruebas sencillas como tinciones) para orientar la terapia y ajustar el espectro de los antimicrobianos. Otro papel para considerar del laboratorio es la medición de concentraciones séricas de antimicrobianos para optimizar la prescripción, permitiendo ajustar la dosis del fármaco en pacientes con condiciones especiales.

- Monitorización: se recomienda establecer indicadores para cuantificar el consumo de antimicrobianos, p ej., días de tratamiento o dosis definida diaria. Además, se sugiere realizar la estimación de costos respecto a la prescripción. Estos indicadores permitirán establecer de forma ulterior la utilidad de los programas de uso racional de antimicrobianos.

- Valorar prescripciones: se recomienda evaluar la indicación del antimicrobiano, la dosis prescrita y su ajuste a condiciones especiales que comprometan la farmacocinética, el riesgo de interacciones medicamentosas, la duración de la terapia y el ajuste de acuerdo con los hallazgos microbiológicos.

Las recomendaciones generales para el uso racional de antimicrobianos se extienden a las recomendaciones para el uso racional de antifúngicos; empero la experiencia reportada en la literatura médica es menor que con antibacterianos.

Reforzando la idea de la necesidad de educación como primer paso en la configuración de programas de uso racional de antifúngicos, se realizó una encuesta a personal médico en diferentes áreas de una institución universitaria en España ${ }^{32}$, preguntando aspectos sobre diagnóstico y tratamiento de infecciones fúngicas especialmente CI y AI. Se encontró que 55\% de los médicos encuestados lograba diferenciar infección de colonización con respecto a aislamiento de Candida spp. en orina y 33\% en muestras respiratorias; $26 \%$ conocía las indicaciones para inicio de anfotericina B liposomal en EFI. Treinta por ciento consideró que anfotericina B liposomal era el tratamiento de elección en AI, y 26,5\% que era de elección en CI con aislamiento de Candida resistente a fluconazol. Sólo $31,5 \%$ sabía que voriconazol estaba recomendado como primera línea de tratamiento para AI, de acuerdo a las guías, y 34,5\% conocía el valor diagnóstico del antígeno galactomanano. Lo que pone en evidencia la gran oportunidad de aprendizaje y la necesidad de estrategias de educación en la aproximación a la EFI y el uso adecuado de antifúngicos.

\section{Experiencias con programas de uso racional de antifúngicos}

Se encuentran pocas publicaciones en la literatura médica mostrando el efecto de la implementación de programas para el uso racional de antifúngicos. Se presenta a continuación el resumen de algunos de los estudios encontrados.

En un estudio prospectivo llevado a cabo en los hospitales de la Universidad de Niza (Francia) ${ }^{4}$, se evaluó el efecto de la implementación de un grupo de uso racional de antifúngicos. Dentro de las intervenciones se describen: registro electrónico de historias clínicas, disponibilidad de pruebas diagnósticas (antígeno galactomanano de Aspergillus, RPC en tiempo real para Aspergillus y RPC panfúngica, determinación de concentraciones plasmáticas de voriconazol o posaconazol, sesiones de educación con el grupo de hematología, formatos para inicio de antifúngicos de alto costo, desarrollo de guías locales, 
creación de un grupo multidisciplinario de manejo de antifúngicos (encargado de hacer auditoría de las prescripciones, y dar recomendaciones y retroalimentación al médico tratante), comunicación telefónica inmediata de los hallazgos desde el laboratorio al médico tratante, disponibilidad de especialista en enfermedades infecciosas permanente al llamado, y acceso a TAC de manera urgente. En 54\% de las prescripciones fue necesario hacer retroalimentación al tratante, y de éstas $88 \%$ fue acatada y realizada. Se logró aumentar el número de tomografías realizadas a los pacientes con sospecha de AI, se disminuyó la terapia combinada por sospecha de AI, hubo una tendencia a tener mayor número de desenlaces favorables en el tratamiento de AI. Con respecto a CI mejoraron la adherencia al inicio de tratamiento de primera línea recomendado, a la duración del tratamiento, a la toma de hemocultivos de control y hubo una tendencia a tener mayor frecuencia de desenlaces favorables. El uso y los costos de los antifúngicos se mantuvieron en el tiempo (seguimiento años 2003 - 2010) a pesar del aumento en la indicación de antifúngicos de mayor costo con el paso de los años.

En el Hospital Puerta de Hierro en Madrid (España), se creó un programa de uso racional de antifúngicos a partir del comité de infecciones, liderado por un grupo compuesto por especialistas en enfermedades infecciosas, farmacia, farmacólogo, especialista en prevención y microbiólogo. Se revisaron las prescripciones de equinocandinas, anfotericina B y voriconazol. Se dieron recomendaciones en $40 \%$ de las prescripciones: cambiar el antifúngico (con adhesión de 37\%), suspender el antifúngico (adhesión de $60 \%$ ), retirar uno de los antifúngicos que se estaba usando en terapia combinada (adhesión de $20 \%$ ), en un caso se recomendó cambiar la vía de administración (sin adhesión). El uso de antifúngicos durante la observación se redujo de 5,06 DDD/100 pacientes-día a 2,92 DDD/100 pacientes-día. La mortalidad de los pacientes tratados de acuerdo con las recomendaciones del programa de uso racional de antifúngicos no tuvo diferencias significativas comparada con la de pacientes sin modificaciones en la terapia ( 17 vs $30 \%, p=0,393$ )

Se realizó un estudio observacional prospectivo con 12 meses de seguimiento en los hospitales de la Universidad de Cambridge (Reino Unido) ${ }^{2}$, evaluando la aplicación de un programa de uso racional de antifúngicos en esa institución. Se realizaron intervenciones clínicas (recomendaciones sobre el diagnóstico o tratamiento de la infección fúngica, o la realización de pruebas diagnósticas adicionales) y financieras (cambio a terapia de menor costo o suspender la terapia). Se dieron recomendaciones clínicas a $64,8 \%$ de las formulaciones de anfotericina $\mathrm{B}$ liposomal, $27,3 \%$ de caspofungina, $88,2 \%$ de micafungina, y 78,7\% de voriconazol. Se recomendó cambio de antifúngico o suspender terapia en $40,8 \%$ de pacientes a quienes se les prescribió anfotericina B, 18\% con prescripción de voriconazol, $77,8 \%$ con caspofungina y $64,7 \%$ de los que recibieron micafungina. Se disminuyó el costo de esos cuatro antifúngicos en $£ 178.708$ en el período de un año.

En el Hospital 12 de Octubre de Madrid (España) ${ }^{33}$, se realizó un estudio de antes-después evaluando el consumo de antifúngicos con posterioridad al inicio de un programa de uso racional de antifúngicos consistente en la revisión de las prescripciones a diario por una persona del grupo de enfermedades infecciosas, además de reuniones a diario con el laboratorio de microbiología para el reporte de aislados fúngicos con relevancia clínica. Se daban recomendaciones directamente al médico tratante sobre la indicación, dosis, vía de administración o cambio de agente antifúngico y se evaluaron las prescripciones de voriconazol, caspofungina y anfotericina B. Se le hizo recomendaciones a $29 \%$ de las prescripciones: $15 \%$ cambio de vía endovenosa a vía oral, $8 \%$ suspender el antifúngico, $6 \%$ cambio a fluconazol. Sólo $1 \%$ de las recomendaciones no fueron aceptadas por el tratante. Se encontró una reducción de 31,4 y 20,2\% en la DDD de voriconazol y caspofungina, respectivamente, pero se observó un incremento de $13,9 \%$ en la DDD de anfotericina B liposomal y de 8,2\% de voriconazol oral. Se logró una reducción de costos en antifúngicos de $11,8 \%$, equivalente para ese momento a USD\$370.681,78.

En un centro hospitalario regional en Lille (Francia) ${ }^{34}$, se evaluó el efecto de la implementación de estrategias de uso racional de antifúngicos como desarrollo y aplicación de guías, el uso obligatorio de formatos de prescripción inicial de antifúngicos (con necesidad de diligenciar un nuevo registro a los siete días de tratamiento) y la visita, dos veces por semana, al servicio de hematología, de un infectólogo para revisar los pacientes con sospecha de infección. Además, se realizaron actividades educativas con los residentes y se planteó el manejo de EFI con árboles de decisiones. En un seguimiento a 10 años, se encontró una reducción superior a $40 \%$ en las prescripciones de antifúngicos (de $1100 \mathrm{DDD}$ /pacientes-día a 600 DDD/pacientes-día) en el servicio de hematología, y de 400 a 300 DDD/pacientes-día en otros servicios.

En el Hospital Universitario Gregorio Marañón en Madrid (España) ${ }^{35}$, se diseñó un programa de uso racional de antifúngicos que incluyó la creación de un grupo interdisciplinario en micosis que evaluaba la epidemiología de las infecciones fúngicas, sugería intervenciones terapéuticas y de prevención, y desarrollaba guías de manejo local. Se desarrolló un sistema computarizado para identificar las nuevas prescripciones de antifúngicos por el grupo de farmacia y emitir alarmas al prescriptor sobre potenciales interacciones farmacológicas y costos; además, requería la justificación de la indicación de acuerdo con las guías locales. En el segundo año de 
intervención se realizó la auditoría de las prescripciones por parte de especialistas en enfermedades infecciosas y se discutieron los casos de manera individual con el médico tratante. También se evaluó el conocimiento de los médicos sobre el diagnóstico y manejo de la EFI. El $40,8 \%$ de las prescripciones requirieron recomendaciones con respecto al diagnóstico (realización de hemocultivos, estudios de serología o imagenología). En el seguimiento a un año de las intervenciones, se identificó una reducción en los costos de $13,9 \%$, y en el segundo año de $12,7 \%$ con respecto al primero; esto fue equivalente a un ahorro de USD\$407.633 y USD\$824.458, respectivamente. En cuanto al consumo de antifúngicos, se logró una disminución de 66,4 a 54,8 DDD/1000 pacientes-día (reducción de $17,5 \%$ ). La mortalidad relacionada a la candidemia pasó de $28 \%$ en 2010 a 16,4\% en 2013.

En el centro médico Vidant de Greenville (Carolina el Norte, E.U.A) ${ }^{36}$, se evaluó el efecto, luego de 13 años de implementación, de un programa de uso racional de antimicrobianos, en el que se incluyeron algunos antifúngicos. Un grupo que articuló un especialista en enfermedades infecciosas con el grupo de farmacia, definió algunos antimicrobianos para ser restringidos y requerir de aval previo por el grupo de uso prudente de antimicrobianos para poder ser despachados por la farmacia. Además, se crearon formatos para la formulación de medicamentos restringidos o controlados por farmacia, que posteriormente se ingresaron al sistema de historias clínicas electrónicas de la institución en forma de alerta, para ser identificados y seguidos por el grupo de farmacia. Igualmente se utilizaban alertas para informarle al médico tratante que el grupo de uso racional de antimicrobianos había emitido alguna recomendación. Se regularon anfotericina B (deoxicolato y complejo lipídico), itraconazol, posaconazol, voriconazol y micafungina. El consumo de antifúngicos luego de 13 años de implementación del programa, se redujo en $71 \%$ y pasó de $151,9 \mathrm{DDD} / 1.000$ pacientes-día a $44 \mathrm{DDD} / 1.000$ pacientes-día.

Como se presenta en esta revisión de la literatura científica, la implementación de medidas para el uso racional de antifúngicos en las diferentes instituciones muestra un impacto favorable en la disminución del consumo de algunos antifúngicos, la optimización en el diagnóstico de la EFI y en algunos desenlaces clínicos.

La implementación de programas para el uso racional de antifúngicos es una necesidad a nivel local y regional; su diseño y puesta en marcha deberán adaptarse al comportamiento epidemiológico de la EFI en cada institución (pacientes en riesgo, microbiología, estrategias de profilaxis), a los recursos (económicos, tecnológicos, humanos) y a la cultura de prescripción apropiada de antifúngicos identificada. En esta revisión no se identificaron experiencias o intervenciones de programas para el uso optimizado de antifúngicos reportados en América Latina.

\section{Conclusión}

El número creciente de pacientes en riesgo de desarrollar EFI ha generado un aumento en la prescripción de antifúngicos, principalmente de forma empírica, por lo que la prescripción inapropiada de este grupo de antimicrobianos es una realidad en la práctica clínica actual. Se hace necesario entonces establecer medidas para el uso óptimo de antifúngicos que pretendan mejorar los desenlaces clínicos, disminuir el riesgo de eventos adversos (propios de los fármacos o por interacciones medicamentosas) y disminuir los costos para el sistema de salud. Las estrategias recomendadas para la implementación de programas para el uso racional de antimicrobianos son aplicables al uso de antifúngicos, teniendo como intervenciones fundamentales para esto la educación y el desarrollo de guías de diagnóstico y tratamiento de EFI a nivel local, implementarlas permitirá lograr los objetivos clínicos y económicos, ajustados al escenario de cada institución y teniendo en cuenta la epidemiología local. Es importante vincular a participantes de diferentes disciplinas para el diseño, la aplicación y el mejoramiento de los programas. El impacto de las intervenciones debe ser cuantificado para retroalimentar los programas y para poder compararse con otras instituciones.

\section{Resumen}

La enfermedad fúngica invasora (EFI) es una entidad que afecta pacientes inmunocomprometidos y críticamente enfermos. En los últimos años, el número de pacientes con riesgo de presentarla viene en aumento, con el consecuente incremento de la formulación de antifúngicos de manera profiláctica, anticipada o empírica. Algunos estudios que evaluaron el uso adecuado de antifúngicos han mostrado que hasta $72 \%$ de las formulaciones pueden ser inapropiadas, exponiendo a los pacientes al riesgo de efectos adversos e interacciones medicamentosas, con mayores costos de la atención. Se han recomendado diferentes intervenciones para el control y el uso racional de antimicrobianos, conocidas como "antimicrobial stewardship", las que se pueden aplicar al uso de antifúngicos denominándose "antifungal stewardship". Se presenta una revisión de la literatura médica sobre el uso apropiado de antifúngicos y el impacto de la implementación de programas de optimización del uso de estos medicamentos en algunos centros. 


\section{Referencias bibliográficas}

1.- Muñoz P, Valerio M, Vena A, Bouza E. Antifungal stewardship in daily practice and health economic implications. Mycoses 2015; 58: 14-25. doi:10.1111/myc. 12329.

2.- Micallef C, Aliyu S H, Santos R, Brown N M, Rosembert D, Enoch D A. Introduction of an antifungal stewardship programme targeting high-cost antifungals at a tertiary hospital in Cambridge, England. J Antimicrob Chemother. February 2015; 2015 (6): 1908-11. doi:10.1093/ $\mathrm{jac} / \mathrm{dkv} 040$.

3.- Ramos A, Pérez-Velilla C, Asensio A, RuizAntorán B, Folguera C, Cantero M, et al. Antifungal stewardship in a tertiary hospital. Rev Iberoam Micol 2015; 32(4): 209-13. doi:10.1016/j.riam.2014.11.006.

4.- Mondain V, Lieutier F, Hasseine L, GariToussaint M, Poiree M, Lions C, et al. A 6-year antifungal stewardship programme in a teaching hospital. Infection. 2013; 41 (3): 6218. doi:10.1007/s15010-013-0431-1.

5.- de Souza MCP, dos Santos AG, Reis AMM. Drug utilization study of systemic antifungal agents in a Brazilian tertiary care hospital. Int J Clin Pharm. 2016; 38 (6): 1398-406 doi:10.1007/s11096-016-0382-6.

6.- Kurosawa M, Yonezumi M, Hashino S, Tanaka J, Nishio M, Kaneda M, et al. Epidemiology and treatment outcome of invasive fungal infections in patients with hematological malignancies. Int J Hematol 2012; 96 (6): 74857. doi:10.1007/s12185-012-1210-y.

7.- Perfect J R, Hachem R, Wingard J R. Update on epidemiology of and preventive strategies for invasive fungal infections in cancer patients. Clin Infect Dis 2014; 59 (suppl 5): S352-S355. doi:10.1093/cid/ciu639.

8.- Nucci M, Garnica M, Gloria A B, Lehugeur D S, Dias V C, Palma L C, et al. Invasive fungal diseases in haematopoietic cell transplant recipients and in patients with acute myeloid leukaemia or myelodysplasia in Brazil. Clin Microbiol Infect 2013; 19 (8): 745-51. doi:10.1111/1469-0691.12002.

9.- Quindós S, Eraso E. Importancia actual de la aspergilosis invasora. En: Pontón J, ed. Guía de Bolsillo de La Aspergilosis Invasora. 2a ed. Bilbao: Revista Iberoamericana de Micología / Asociación Española de Micología; 2008: 3-6.

10.- Hamdy R F, Zaoutis T E, Seo S K. Antifungal stewardship considerations for adults and pediatrics. Virulence 2017; 8 (6): 658-72. doi: 1 $0.1080 / 21505594.2016 .1226721$.

11.- Neofytos D, Lu K, Hatfield-Seung A, Blackford A, Marr K A, Treadway S, et al. Epidemiology, outcomes, and risk factors of invasive fungal infections in adult patients with acute myelogenous leukemia after induction chemotherapy. Diagn Microbiol Infect Dis 2013; 75 (2): 144-9. doi:10.1016/j. diagmicrobio.2012.10.001.

12.- Samanta P, Singh N. Complications of invasive mycoses in organ transplant recipients. Expert Rev Anti Infect Ther 2016; 14 (12): 1195-202. doi:10.1080/14787210.2016.1242412.

13.- Gavaldà J, Meije Y, Fortún J, Roilides E, Saliba F, Lortholary O, et al. Invasive fungal infections in solid organ transplant recipients. Clin Microbiol Infect 2014; 20: 27-48. doi:10.1111/1469-0691.12660.

14.- Dhillon R H, Clark J. Fungal infections in the critically ill. Trends Anaesth Crit Care 2011; 1 (4): 210-8. doi:10.1016/j.tacc.2011.05.007.

15.- De Pauw B, Walsh T J, Donnelly J P, Stevens D A, Edwards J E, Calandra T, et al. Revised definitions of invasive fungal disease from the European Organization for Research and Treatment of Cancer/Invasive Fungal Infections Cooperative Group and the National Institute of Allergy and Infectious Diseases Mycoses Study Group (EORTC/MSG) C. Clin Infect Dis 2008; 46 (12): 1813-21. doi: $10.1086 / 588660$.

16.- Aitken S L, Beyda N D, Shah D N, Palmer H R, Lasco T M, Koo H, et al. Clinical practice patterns in hospitalized patients at risk for invasive candidiasis. Ann Pharmacother 2014; 48 (6): 683-90. doi: $10.1177 / 1060028014529928$

17.- Vazin A, Davarpanah M, Ghalesoltani S. Antifungal agent utilization evaluation in hospitalized neutropenic cancer patients at a large teaching hospital. Drug Health Patient Saf 2015; 97. doi:10.2147/DHPS.S80762.

18.- Valerio M, Vena A, Bouza E, Reiter N, Viale P, Hochreiter M, et al. How much European prescribing physicians know about invasive fungal infections management? BMC Infect Dis $2015 ; 15$ (1): 80 . doi: $10.1186 / \mathrm{s} 12879-015$ 0809-z.

19.- Ibrahim O M, Polk R E. Antimicrobial use metrics and benchmarking to improve stewardship outcomes. Infect Dis Clin North Am 2014; 28 (2): 195-214. doi:10.1016/j. idc.2014.01.006.

20.- Fondevilla E, Grau S, Mojal S, Palomar M, Matas L, Gudiol F. Consumption of systemic antifungal agents among acute care hospitals in Catalonia (Spain), 2008-2013. Expert Rev Anti Infect Ther 2016; 14 (1): 137-44. doi:10.1586/1 4787210.2016.1096776.

21.- Ramírez E, García-Rodríguez J, Borobia A M, Ortega J M, Lei S, Barrios-Fernández A, et al. Use of antifungal agents in pediatric and adult high-risk areas. Eur J Clin Microbiol Infect Dis 2012; 31 (3): 337-47. doi:10.1007/s10096-0111315 -x.

22.- Van Boeckel T P, Gandra S, Ashok A, Caudron Q, Grenfell B T, Levin S A, et al. Global antibiotic consumption 2000 to 2010: an analysis of national pharmaceutical sales data. Lancet Infect Dis 2014; 14 (8): 742-50. doi:10.1016/S1473-3099 (14)70780-7.
23.- Kim S H, Shin J H, Kim E-C, Lee K, Kim M $\mathrm{N}$, Lee $\mathrm{W}$ G, et al. The relationship between antifungal usage and antifungal susceptibility in clinical isolates of Candida: a multicenter Korean study. Med Mycol 2009; 47 (3): 296304. doi:10.1080/13693780802291445.

24.- HOF H. Is there a serious risk of resistance development to azoles among fungi due to the widespread use and long-term application of azole antifungals in medicine? Drug Resist Updat 2008; 11 (1-2): 25-31. doi:10.1016/j. drup.2008.01.001.

25.- Alvarez-Moreno C, Lavergne R-A, Hagen F, Morio F, Meis J F, Le Pape P. Azole-resistant Aspergillus fumigatus harboring TR34/L98H, TR46/Y121F/T289A and TR53 mutations related to flower fields in Colombia. Sci Rep. 2017; 7: 45631. doi:10.1038/srep45631.

26.- Pollack L A, Srinivasan A. Core elements of hospital antibiotic stewardship programs from the Centers for Disease Control and Prevention. Clin Infect Dis 2014; 59 (supp1_3): S97-S100. doi:10.1093/cid/ciu542.

27.- Rodríguez-Baño J, Paño-Pardo JR, AlvarezRocha L, Asensio A, Calbo E, Cercenado E, et al. Programas de optimización de uso de antimicrobianos (PROA) en hospitales españoles: documento de consenso GEIHSEIMC, SEFH y SEMPSPH. Enferm Infecc Microbiol Clin 2012; 30 (1): 22.e1-22.e23. doi:10.1016/j.eimc.2011.09.018.

28.- Barlam T F, Cosgrove S E, Abbo L M, MacDougall C, Schuetz A N, Septimus E J, et al. Implementing an Antibiotic Stewardship Program: Guidelines by the Infectious Diseases Society of America and the Society for Healthcare Epidemiology of America. Clin Infect Dis 2016; 62 (10): e51-e77. doi:10.1093/ cid/ciw118.

29.- Nagel J L, Kaye K S, LaPlante K L, Pogue J $M$. Antimicrobial stewardship for the infection control practitioner. Infect Dis Clin North Am 2016; 30 (3): 771-84. doi:10.1016/j. idc.2016.04.012.

30.- López-Medrano F, Moreno-Ramos F, de Cueto M, Mora-Rillo M, Salavert M. How to assist clinicians in improving antimicrobial prescribing: Tools and interventions provided by stewardship programs. Enferm Infecc Microbiol Clin 2013; 31: 38-44. doi:10.1016/ S0213-005X(13)70131-9.

31.- Arvanitis M, Anagnostou T, Fuchs B B, Caliendo A M, Mylonakis E. Molecular and nonmolecular diagnostic methods for invasive fungal infections. Clin Microbiol Rev 2014; 27 (3): 490-526. doi:10.1128/CMR.00091-13.

32.- Valerio M, Muñoz P, Rodríguez-González C, Sanjurjo M, Guinea J, Bouza E. Training should be the first step toward an antifungal stewardship program. Enferm Infecc Microbiol Clin 2015; 33 (4): 221-7. doi:10.1016/j. eimc.2014.04.016. 
33.- López-Medrano F, Juan R S, Lizasoain M, Catalán M, Ferrari J M, Chaves F, et al. A non-compulsory stewardship programme for the management of antifungals in a university-affiliated hospital. Clin Microbiol Infect 2013; 19 (1): 56-61. doi:10.1111/j.14690691.2012.03891.x.

34.- Alfandari S, Berthon C, Coiteux V. Antifungal stewardship: Implementation in a French teaching hospital. Médecine Mal Infect 2014; 44 (4): 154-8. doi:10.1016/j. medmal.2014.01.012.

35.- Valerio M, Muñoz P, Rodríguez C G, Caliz B, Padilla B, Fernández-Cruz A, et al. Antifungal stewardship in a tertiary-care institution: a bedside intervention. Clin Microbiol Infect
2015; 21 (5): 492.e1-492.e9. doi:10.1016/j. cmi.2015.01.013.

36.- Cook P P, Gooch M. Long-term effects of an antimicrobial stewardship programme at a tertiary-care teaching hospital. Int J Antimicrob Agents. 2015; 45 (3): 262-7. doi:10.1016/j. ijantimicag.2014.11.006.

37.- Sutepvarnon A, Apisarnthanarak A, Camins B, Mondy K, Fraser V J. Inappropriate use of antifungal medications in a tertiary care center in Thailand: a prospective study. Infect Control Hosp Epidemiol 2008; 29(04): 370-3. doi:10.1086/587633.

38.- Nivoix Y, Launoy A, Lutun P, Moulin J C, Phai Pang K A, Fornecker LM, et al. Adherence to recommendations for the use of antifungal agents in a tertiary care hospital. $\mathrm{J}$ Antimicrob Chemother 2012; 67 (10): 2506-13. doi:10.1093/jac/dks256.

39.- Fabien L, Foroni L, Brion J-P, Maubon D, Stahl J-P, Pavese P. Évaluation de la prescription des antifongiques dans un centre hospitalier universitaire: encore trop de prescriptions inadaptées malgré des actions de formation. Presse Med. 2014; 43 (9): e241-e250. doi: 10.1016/j. lpm.2013.11.029.

40.- Abdel Fattah M, Demoré B, Girardeau A, Heit S, May T, Rabaud C, et al. Antifungal agents use in a French administrative region. Médecine Mal Infect 2015; 45 (7): 279-85. doi:10.1016/j.medmal.2015.04.010. 